\title{
Una mirada praxeológica de la experiencia investigativa desarrollada en torno al Proyecto "La Caracterización de las prácticas docentes de la Facultad de Educación de UNIMINUTO"1
}

\author{
Nadia Paola Acosta Marroquín ${ }^{2}$ \\ Universidad Libre, Bogotá, Colombia \\ paolitaj3@gmail.com \\ Zaily Del Pilar García Gutiérrez ${ }^{3}$ \\ Corporación Universitaria Minuto de Dios -UNIMINUTO, Bogotá, Colombia \\ zaily.garcia@gmail.com \\ Benjamín Barón Velandia ${ }^{4}$ \\ benjaminbv@gmail.com
}

1 Esta publicación es resultado de la investigación iniciada y concluida en el 2015, titulada: "Caracterización de las prácticas pedagógicas de los docentes de la Facultad de Educación de la Corporación Universitaria Minuto de Dios-UNIMINUTO" con el objetivo "Caracterizar las prácticas docentes en los programas de pregrado y posgrado de la Facultad de Educación de la Corporación Universitaria Minuto de Dios-UNIMINUTO". Investigación financiada y ejecutada por la Corporación Universitaria Minuto de Dios-UNIMINUTO.

2 Licenciada en Pedagogía Infantil de la Universidad Distrital Francisco José de Caldas. Magister en Investigación Social Interdisciplinaria de la Universidad Distrital Francisco José de Caldas. DocenteInvestigadora en la Facultad Ciencias de la Educación.

3 Magister en Comunicación Educativa de la Universidad Tecnológica de Pereira. Doctoranda en Educación: Currículum, profesorado e instituciones educativas de la Universidad de Granada España. Docente-Investigadora en la Facultad de Educación.

4 Candidato a Doctor en Educación de la Universidad Central de Nicaragua. Docente-Investigador en la Facultad de Educación. Corporación Universitaria Minuto de Dios- UNIMINUTO. Fundador y Vicepresidente de la Fundación para la Calidad Educativa-FUCAED. 


\title{
Una mirada praxeológica de la experiencia investigativa desarrollada en torno al Proyecto "La Caracterización de las prácticas docentes de la Facultad de Educación de UNIMINUTO”
}

\section{Resumen}

Las prácticas docentes en Educación Superior se han convertido en un interés creciente en la comunidad académica dado el impacto que tienen en la formación de los futuros profesionales. Especialmente, las facultades de educación se ven convocadas a reflexionar sobre dicha práctica como punto de partida para su cualificación; esto en el marco de la reciente reestructuración emitida por el Ministerio de Educación Nacional que pretende optimizar la formación de profesores en Colombia. La Facultad de Educación de la Corporación Universitaria Minuto de Dios-UNIMINUTO, sintonizada con estas necesidades y coherente con su misión institucional, desarrolla el proyecto de Investigación "Caracterización de las prácticas docentes de la Facultad de Educación de la Corporación Universitaria Minuto de Dios- UNIMINUTO". Éste es una experiencia investigativa en el marco de la praxeología que se convierte en una valiosa oportunidad de aprendizaje tanto para los docentes invitados, participantes de la investigación, como para el equipo de docentes investigadores.

Palabra clave: Praxeología, práctica docente, docencia universitaria, formación docente.

\section{A praxeological look to research experience developed around the project "Characterization of the teaching practices of the Faculty of Education UNIMINUTO"}

\begin{abstract}
The teaching practices of university teaching have acquired a growing interest in the academic community, given its impact on the formation of future professionals. Particularly the education faculties are gathered to reflect about this practice as a starting point for qualification; all this within the context of the recent restructuring issued by the Ministry of National Education to ensure the optimization of the formation of teachers in Colombia. The Faculty of Education of the Corporación Universitaria Minuto de Dios -in accordance with these needs and consistently with its institutional mission - developed the research project "Characterization of the teaching practices of the Faculty of Education of the Corporación Universitario Minuto de Dios-UNIMINUTO,"; this consist of a research experience within the framework of praxeology, which will become a valuable learning opportunity for both guest lecturers and research subjects, as well as for the team of research teachers.
\end{abstract}

Keywords: Praxeology, teaching practice, university teaching, teacher's formation.

\section{Uma mirada praxeológica da experiência investigativa desenvolvida em torno do Projeto "A Caracterização das práticas docentes da Faculdade de Educação da UNIMINUTO".}

\section{Resumo}

As práticas docentes em Educação Superior converteram-se em um interesse crescente na comunidade acadêmica, dado o impacto que têm na formação dos futuros profissionais. Especialmente as Faculdades de Educação, veem-se convocadas a reflexionar sobre dita prática como ponto de partida para sua qualificação; isto no marco da recente reestruturação emitida pelo Ministério de Educação Nacional que pretende aperfeiçoar a formação de professores na Colômbia. A Faculdade de Educação da Corporação Universitária Minuto de Deus (UNIMINUTO), sintonizada com estas necessidades e coerente com sua missão institucional, desenvolve o projeto de Investigação "Caracterização das práticas docentes da Faculdade de Educação da Corporação Universitária Minuto de Deus" (UNIMINUTO), experiência investigativa no marco da praxeologia, converte-se em uma valiosa oportunidade de aprendizagem tanta para os docentes convidados como participantes da investigação, como para a equipe de docentes investigadores.

Palavra-chave: Praxeologia, prática docente, docência universitária, formação docente. 


\section{Introducción}

El presente artículo tiene por objetivo generar un escenario de divulgación y socialización sobre el proceso Praxeológico vivenciado en el marco de una investigación llevada a cabo en la Facultad de Educación de UNIMINUTO durante el 2015 en adelante, Ilamada FEDU. Este ejercicio pretende recrear la experiencia praxeológica suscitada por la participación de un equipo interdisciplinario de docentes, en la investigación titulada "Caracterización de las prácticas docentes de la Facultad de Educación de la Corporación Universitaria Minuto de DiosUNIMINUTO", uno de los proyectos financiados por la IV Convocatoria para el desarrollo y fortalecimiento de la investigación en UNIMINUTO; cuya naturaleza investigativa se fundamenta justamente en la investigación praxeológica. Se inicia haciendo una presentación general del contexto que sitúa la problemática en la cual surge el proyecto, dando paso a una breve presentación de la estructura general del proyecto para, finalmente, disertar desde el enfoque praxeológico respecto a las experiencias vividas por los investigadores, oportunidades de aprendizajes y demás dinámicas de cambio que han caracterizado esta investigación.

\section{La Facultad de Educación, un escenario dinámico en el que se gesta la investigación Praxeológica como una oportunidad de cualificación docente}

Caracterizar las prácticas de los docentes de pregrado y posgrado de la Facultad de Educación de la Corporación Universitaria Minuto de Dios se convierte en el primer paso de un proceso proyectado a por lo menos tres o cuatro años. A partir de éste se busca la cualificación de las prácticas del docente universitario encargado de la formación del futuro maestro UNIMINUTO. Este proyecto de investigación, en su planteamiento y desarrollo, encarna fielmente el espíritu Praxeológico que caracteriza a la Facultad de Educación, no sólo por la brindar la oportunidad de reflexionar respecto a la praxis en torno a la docencia universitaria, para movilizar una mejora continua de las práctica, sino porque representa una iniciativa para continuar en la transformación de las docencias en educación superior, en la Facultad de Educación de UNIMINUTO. Asimismo, en todas las facultades del sistema y, ipor qué no?, movilizar la transformación de las prácticas docentes de otras facultades de educación del País.

Desde su surgimiento en 1992, la Facultad de Educación se ha caracterizado por ser pionera en las reflexiones y procesos pedagógicos encaminados al fortalecimiento de la labor formativa del futuro profesional colombiano, con el sello del Minuto de Dios; revelándose como un escenario inquieto y propositivo que busca innovaciones educativas comprometidas con la realidad social colombiana a partir de una de 
las más reconocidas: la Pedagogía Praxeológica. En este sentido, específicamente desde su disciplina, siempre se ha comprometido con garantizar la formación de maestros, constructores de conocimiento pedagógico y transformadores de lo social. Al respecto, la FEDU-UNIMINUTO (2012) por medio del Proyecto Pedagógico de la Facultad de Educación expresa:

UNIMINUTO quiere formar profesionales que sean al mismo tiempo innovadores sociales; para ello creemos que la aparición de una nueva sociedad sólo es posible cuando se comprende que las relaciones sociales se constituyen en un tiempo y espacio determinado, cuando se entiende que en la cotidianidad se constituye saber, que la educación y la cultura son procesos en continua transformación, y que la práctica, siempre aunada a la teoría, es fuente de aprendizaje y de conocimiento. Todos ellos son principios fundamentales de nuestro modelo educativo: la pedagogía praxeológica (p. 20).

En coherencia con lo anterior, año tras año, la FEDU ha generado y posibilitado una serie de proyectos de investigación que hacen de ésta una de las principales creadoras, gestoras y divulgadoras de la Praxeología, la cual siempre invita a todos los actores educativos vinculados en la institución a observar (Ver), profundizar teóricamente (Juzgar), diseñar e implementar planes de mejora (Actuar) y reflexionar, evaluar y transformar sobre lo ocurrido como principales estrategias de aprendizajes (Devolución Creativa) (Juliao, 2011, 2014). De manera general, se trata de observar, reflexionar analíticamente, ajustar la acción de manera más pertinente y evaluar lo ocurrido para aprender; todo en busca de la transformación de las prácticas de los docentes, que necesariamente redundan en beneficio de las transformaciones sociales porque son relaciones interdependientes "prácticas docentes"-"prácticas sociales".

Fruto de todo este trasegar, la Facultad de Educación traza un plan estratégico (2013-2019) denominado "Escuelas pertinentes en pedagogías e innovaciones Sociales en Educación". Éste es considerado como eje integrador y articulador de la docencia, la investigación, la responsabilidad social y la internacionalización, que busca identificar, apropiar e implementar los criterios orientadores de la pertinencia en el campo de las pedagogías y las innovaciones sociales y su incidencia en las gestiones académicas y administrativas de la Facultad de Educación. Su principal objetivo está dirigido a consolidar la comunidad educativa de la Facultad de Educación para cualificar las condiciones académicas y administrativas encaminadas al logro de la pertinencia, en el campo de las pedagogías y las innovaciones sociales.

En este orden ideas, la investigación titulada "Caracterización de las prácticas docentes de la Facultad de Educación de la Corporación Universitaria Minuto de Dios-UNIMINUTO" se convierte en una de las alternativas generadas en la y por la 
Una mirada praxeológica de la experiencia investigativa desarrollada en torno al Proyecto

"La Caracterización de las prácticas docentes de la Facultad de Educación de UNIMINUTO"

| Nadia Paola Acosta Marroquín | Zaily Del Pilar García Gutiérrez | Benjamín Barón Velandia |

FEDU para aportar a la consecución de dicho plan estratégico, cuyo cumplimiento garantizará que continúe destacándose por ser una facultad innovadora y propositiva en el escenario de la educación superior. Esta investigación surge como una ruta que permite continuar pensando en las prácticas docentes llevadas a cabo por los programas de pregrado y posgrado, para la formación de futuros docentes desde una perspectiva praxelógica que reflexiona sobre el estado de la docencia universitaria como un lugar llamado a la mejora continua, que se pueda adaptar a las necesidades y desafíos que tiene la cultura contemporánea. Este es un reto inminente no sólo para la Facultad de Educación, sino también para todas la Facultades del Sistema UNIMINUTO, de Bogotá y de Colombia; por lo tanto, la experiencia desarrollada por este proyecto se convierte en una iniciativa que podría vincular escenarios investigativos como la Asociación Colombiana de Facultades de Educación (ASCOFADE) o el mismo Ministerio de Educación (MEN) porque logra responder a las actuales necesidades de fortalecimiento de las Facultades de Educación del país, esto en coherencia con la reciente publicación de los Lineamientos de Calidad para las Facultades de Educación.

\section{La caracterización de las prácticas de los docentes de la FEDU, una apuesta investigativa por el fortalecimiento de la FEDU}

Ante este panorama, desde la postura de una universidad que se reflexiona constantemente para mejorar, que se siente llamada a pensar el proceso de formación que se lleva a cabo con los estudiantes, en coherencia con su modelo praxeológico, y, además, centrando la mirada en sus docentes, se hace fundamental reconocer el papel que éstos desempeñan como principales acompañantes de los procesos formativos. Esta experiencia se concreta en las propuestas formativas mediante el diseño, desarrollo y evaluación de los espacios académicos a su cargo, que tienen como objetivo la formación de profesionales integrales.

En este orden de ideas, la Facultad de Educación requiere docentes universitarios preparados para los diferentes retos pedagógicos en todos los escenarios y campos profesionales, desde una postura praxeológica. Ésta es la encargada de formar al docentes como "un profesional creativo y conocedor del valor que tiene la relación armónica entre la educación y el desarrollo" (García, 2011, p. 198), es decir, agentes sociales responsables de orientar la formación de los ciudadanos. Lo anterior implica hacer una reflexión de la práctica docente y a partir de ello generar procesos de transformación y cualificación de las prácticas docentes de la FEDU. Es por esto que resulta fundamental adelantar un ejercicio de indagación que permita conocer el estado de las prácticas docentes al interior de los programas de pregrado y posgrado de la Facultad de Educación, pues, en este sentido surge el siguiente interrogante: ¿cuáles son las características de las prácticas docentes de los programas de pregrado y posgrado en Facultad de Educación de la Corporación Universitaria Minuto de Dios-UNIMINUTO? 
Caracterizar las prácticas docentes en los programas de pregrado y posgrado de la Facultad de Educación de la Corporación Universitaria Minuto de DiosUNIMINUTO, como objetivo general de esta investigación requiere: describir las prácticas docentes de los programas de pregrado y posgrado de la Facultad de Educación de la Corporación Universitaria Minuto de Dios- UNIMINUTO; reconocer las características y los sentidos de las prácticas docentes emergentes de los programas de pregrado y posgrado de la Facultad de Educación de la Corporación Universitaria Minuto de Dios-UNIMINUTO y establecer las relaciones existentes entre las prácticas docentes de la FEDU con el Modelo Educativo de UNIMINUTO, en coherencia con la Pedagogía Praxeológica como punto de partida para la cualificación de las prácticas docentes.

La propuesta investigativa orientada por el Enfoque Praxeológico propone una metodología abierta, reflexiva y con miras a las transformaciones. Se retoman elementos de La investigación total de Hugo Cerda que en articulación con la praxeología permiten abordar el proceso investigativo desde una mirada amplia, plural y diversa de la realidad social. Esta posición implica una ruptura reflexiva entre los paradigmas cuantitativo y cualitativo y convierte la investigación, como lo propone ese autor "en una experiencia total, integradora y amplia, sin restricciones y limitaciones de ningún tipo" (Cerda, 1994, p.11).

Este proceso de experiencia investigativa se ha ido complejizando y madurando, los encuentros de saberes interdisciplinares y de experiencias docentes han aportado a la construcción y crecimiento de lo que hoy es el proyecto en curso. Así mismo, la oportunidad de vincular estudiantes a semilleros desde esta experiencia investigativa ha ido enriqueciendo la mirada de ésta y aportando a la formación misma de los estudiantes en el proceso investigativo.

\section{Un equipo de docentes investigadores que reflexiona praxeológicamente su experiencia investigativa}

El reconocimiento de un ejercicio investigativo, que permite la reflexión del quehacer de los maestros, es una oportunidad para la transformación de escenarios educativos, donde los docentes tienen como labor cuestionarse permanentemente desde una mirada pedagógica sobre los distintos procesos y realidades de la educación superior. Éste es un reto que han asumido un grupo de profesores de la Facultad de Educación que en coherencia con la praxeología han desarrollado un ver de manera reflexiva, un juzgar desde una perspectiva crítica, un actuar coherente con los discursos y una devolución creativa que posibilita procesos de mejora y transformación a partir de una experiencia investigativa y reflexiva en donde se constituyen como sujetos investigadores. 
Una mirada praxeológica de la experiencia investigativa desarrollada en torno al Proyecto

"La Caracterización de las prácticas docentes de la Facultad de Educación de UNIMINUTO"

| Nadia Paola Acosta Marroquín | Zaily Del Pilar García Gutiérrez | Benjamín Barón Velandia |

Es interesante cómo desde este proceso de investigación se promueve el desarrollo profesional de docente universitario praxeológico, en el que se invita a la "constante reflexión, analítica y propositiva, del quehacer (...) quien busca observar críticamente y evaluar su praxis, con el fin de proponer otras alternativas, mucho más pertinentes al momento de desarrollar los procesos de formación" (Acosta y García, 2013, p. 503). Lo anterior, no sólo para dar cuenta de un proceso investigativo, sino que desde el mismo ejercicio trasciendan al quehacer propio de sus prácticas docentes y su formación misma. Esta característica posibilita la comprensión de una investigación más flexible en términos de romper con la tradición del objetivismo y se instala en el paradigma emergente de la complejidad que encuentra en el enfoque biográfico narrativo (Bolívar, 2002) un escenario propicio para develar las realidades de los sujetos que desarrollan las prácticas docentes trascendiendo el acto de "dar clase" (Baron, et.al., 2012). La organización de un equipo intergeneracional e interdisciplinario ha permitido el encuentro de saberes y la consolidación de un grupo de estudio donde crecen los debates y las reflexiones de la experiencia.

Esta práctica ha generado que los docentes que participan en este espacio fortalezcan sus procesos de lectura, de interpretación y argumentación, poniendo sobre la mesa distintos debates desde las construcciones epistemológicas y posturas metodológicas desarrolladas a partir de la investigación, reconociendo, además, los procesos de co-construcción de los aprendizajes. Nadie aprende en la individualidad, sino en las redes de interrelaciones existentes entre las diversas disciplinas y experiencias que se convertían para unos y otros en interaprendizajes (Baron y Cancino, 2014), (Assmann, 2002). Esto en coherencia con el papel fundamental que tiene la investigación en la educación superior y, en este caso, la oportunidad de transformación que tienen los docentes desde esta experiencia investigativa colectiva. Por otro lado, este proceso incide de manera reflexiva y crítica en los participantes de la investigación quienes, junto con otros, se miran a ellos mismos y a sus prácticas docentes; lo cual permite interpretar y juzgar lo que están haciendo, cómo día a día se constituyen como maestros, para desde allí transformar sus acciones y proponer cambios pedagógicos que aporten a la mejora de la Educación Superior.

Este proyecto de investigación trasciende una posible salida a la reciente publicación de los Lineamientos de Calidad para las Licenciaturas en Educación, por parte del Ministerio de Educación Nacional (MEN), donde se estandarizan una serie de exigencias encaminadas a la formación de docentes integrales y competentes que ejerzan la profesión como agentes activos de transformación, comprometidos con la búsqueda de un país mucho más equitativo justo y democrático. Es por ello que se requieren prácticas docentes cualificadas y pertinentes. Así, el ejercicio fundamental para esta cualificación es comprender las dinámicas pedagógicas que subyacen en el ejercicio de formación. Se trata de adelantar una caracterización de la labor docente orientada al desarrollo profesoral, que propende por la consolidación del proyecto 
personal, profesional y académico. Este fortalecimiento impacta la calidad de los programas ofertados por la institución y en consecuencia aporta a la transformación de las realidades sociales del país.

Este proyecto se configura como un aporte al sector educativo porque propone reflexionar la praxis del quehacer docente al interior de la Facultad de Educación como una alternativa de producción y divulgación de conocimiento, lo cual resulta muy pertinente para robustecer el escenario académico que gira en torno a la Docencia Universitaria en educación; así mismo, favorece la calidad educativa para la formación de los futuros docentes que respondan a las necesidad cambiantes de la sociedad colombiana. Es una oportunidad para reflexionar los elementos del Enfoque Praxeológico en el marco del modelo educativo de UNIMINUTO. En este sentido, conocer el quehacer de los docentes de la FEDU permite reconocer la diversidad de prácticas pedagógicas y su posible resonancia con la Pedagogía Praxeológica. Este proyecto de investigación se convierte en uno de los soportes para desarrollar las metas del plan estratégico 2013-2019 de la FEDU, ya que a partir del reconocimiento de las prácticas docentes aporta a la configuración y consolidación de una escuela pertinente en pedagogías e innovaciones sociales en educación, propósito que contribuirá al posicionamiento de la FEDU como un referente epistemológico para mejorar las prácticas docentes, ya no sólo de la facultad, sino de la Sede principal, del mismo Sistema UNIMINUTO y de otras Instituciones de Educación Superior.

\section{Conclusiones}

Esta es una experiencia investigativa que de acuerdo a su naturaleza praxeológica ha posibilitado aprendizajes constantes por parte de todos los actores vinculados en el proceso. Para los docentes investigadores ha generado diferentes oportunidades para aprender a llevar a cabo una investigación Praxeológica, lo cual ha implicado el fortalecimiento de hábitos de estudios, producción escrita y trabajo colectivo, aprendizajes concretos sobre epistemologías de la investigación y el soporte teórico de la docencia universitaria. A gran parte de los integrantes del equipo les ha significado la incursión por primera vez en la investigación como una de las funciones sustantivas que movilizan la docencia universitaria. Gracias a esto han tenido la oportunidad de participar desde el inicio de la idea, la consolidación de la propuesta, la participación en una convocatoria, la asignación del presupuesto, la ejecución y cierre del proyecto, junto con las diferentes estrategias de divulgación y socialización de los resultados obtenidos. Se reconoce el aporte de esta experiencia a la consolidación de la Facultad de Educación como una comunidad académica fortalecida en la que todos sus miembros tienen la oportunidad de mejorar sus condiciones en el marco del desarrollo profesoral y personal, sin generar divisiones o fragmentaciones en su complexión humana. 
Con respecto a los otros docentes involucrados, es decir los participante de la investigación, con quienes se recolectan los datos requeridos para dar cumplimiento al objetivo y así dar respuesta a la problemática, también es posible evidenciar una serie de valiosos aprendizajes constituidos en el marco de la aplicación de una investigación Praxeológica, en la que se invita al docente participante a reconstruir su práctica mediante un encuentro de saberes, que moviliza un proceso reflexivo. Esto supone el fortalecimiento de su capacidad analítica, crítica, reflexiva y retrospectiva, práctica que ha generado el fortalecimiento de habilidades escriturales para producir un texto que da cuenta de los procesos adelantados. Los docentes vinculados a la investigación son los principales beneficiados ya que son ellos quienes gracias a su activa participación, simultáneamente están transformando y mejorando su prácticas como docentes universitarios.

Es posible que a partir de una mirada particular de una experiencia como la de UNIMINUTO se aborde una reflexión ante el panorama educativo de Colombia, que motive, oriente y movilice otras propuestas pedagógicas acordes con las necesidades y realidades de la educación superior. Desde una postura praxeológica que busca aportar a los cambios de sociedades y comunidades, a partir de la transformación de sujetos desde su propia reflexión "autocrítica". En este caso se hace pertinente incitar y provocar a la reflexión misma de los educadores colombianos que, como gestores de sociedad, tienen la labor de repensar sus prácticas y reinventar propuestas acordes con las demandas de la sociedad y de los jóvenes. El reto es construir comunidad académica y crear redes de conversaciones entre las mismas facultades, que trascendiendo la sede principal, y el sistema UNIMINUTO, Ileguen a las diferentes facultades de educación de la ciudad y del país. 


\section{Referencias}

Acosta, N. y García Zaily (2013). La formación en Investigación de los Pedagogos Infantiles, una apuesta a la transformación social. En: Memorias Congreso de Investigación y Pedagogía IP/ 2013. Tunja: Universidad Pedagógica y Tecnológica de Colombia, 502-513.

Assmann, H. (2002). Placer y ternura en la educación. : Narcea S.A. De Ediciones.

Barón, B. Cancino J., García D. y Guativa J. (2012) Y ellos me hacían sentir muy mal: La comunicación inclusiva, un horizonte de posibilidades. En, Echavarría y Mesa (Ed.) Formación ético política: itinerarios de ciudadanía y violencia escolar. Bogotá: Kimpres.

Barón, B. y Cancino J. (2014) La investigación biográfico-narrativa en educación: un enfoque de investigación co-construido desde las subjetividades, desde el tejido de la multiplicidad de realidades. Praxis pedagógica,15, 89-102.

Bolívar, A. (2002). “¿De nobis ipsis silemus?”: Epistemología de la investigación biográficonarrativa en educación. Revista Electrónica de Investigación Educativa. Recuperado de: http://www.fts.uner.edu.ar/catedras03/tfoi/2010/Bolivar_2002.pdf

Cerda, H. (1994). La investigación Total: Unidad metodológica en la investigación científica. Bogotá: Cooperativa Editorial Magisterio.

FEDU-UNIMINUTO (2012). Proyecto Pedagógico de la Facultad de Educación (PPFE). Bogotá.

García, Z. (2011). La evaluación en la formación del Pedagogo Infantil. Reflexión en torno a una experiencia significativa. En: Políticas y experiencias de evaluación en la Educación Superior en Colombia. Bogotá: Universidad Pedagógica Nacional, 197-208.

Juliao, C. (2014). Una Pedagogía Praxeológica. Bogotá: Corporación Universitaria Minuto de Dios.

Juliao, C. (2011). El Enfoque Praxeológico. Bogotá: Corporación Universitaria Minuto de Dios.

Recibido: 15 de enero de 2015

Aceptado: 20 de marzo de 2015

Como citar: Acosta, N., García, A. y Barón, B. (2015) Una mirada praxeológica de la experiencia investigativa desarrollada en torno al Proyecto "La Caracterización de las prácticas docentes de la Facultad de Educación de UNIMINUTO". Praxis Pedagógica, 17, 15-24 